\title{
Correction to: Retinoic acid receptor $\gamma$ activation promotes differentiation of human induced pluripotent stem cells into esophageal epithelium
}

\author{
Yasufumi Koterazawa ${ }^{1,2,3} \cdot$ Michiyo Koyanagi-Aoi ${ }^{1,2,4} \cdot$ Keiichiro Uehara $^{1,2,5}$. \\ Yoshihiro Kakeji ${ }^{3}$ Takashi Aoi ${ }^{1,2,4}$
}

Published online: 15 September 2020

(C) Japanese Society of Gastroenterology 2020

Correction to: J Gastroenterol (2020) 55:763-774

https://doi.org/10.1007/s00535-020-01695-7

In the original publication of the article, the following errors were noted and corrected in this correction.

In Figure 1b, an unnecessary letter "d" should be deleted.

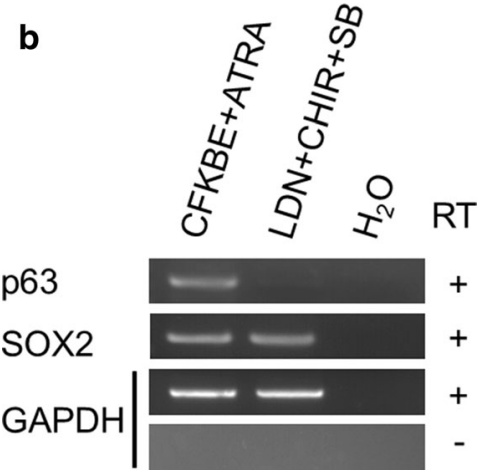

In Figure 4d and the caption of Figure 4e, "LY29953" should be corrected to "LY2955303". In the Figure 4e, "LY29553" and "LY" should be corrected to "LY2955303". The revised figures are given in this correction.

The original article can be found online at https://doi.org/10.1007/ s00535-020-01695-7.

Takashi Aoi

takaaoi@med.kobe-u.ac.jp

1 Division of Advanced Medical Science, Graduate School of Science, Technology and Innovation, Kobe University, 7-5-1 Kusunoki-cho, Chuo-ku, Kobe 650-0017, Japan

2 Department of iPS Cell Applications, Graduate School of Medicine, Kobe University, Kobe, Japan

3 Division of Gastrointestinal Surgery, Department of Surgery, Graduate School of Medicine, Kobe University, Kobe, Japan

4 Center for Human Resource Development for Regenerative Medicine, Kobe University Hospital, Kobe, Japan

5 Division of Pathology, Graduate School of Medicine, Kobe University, Kobe, Japan 

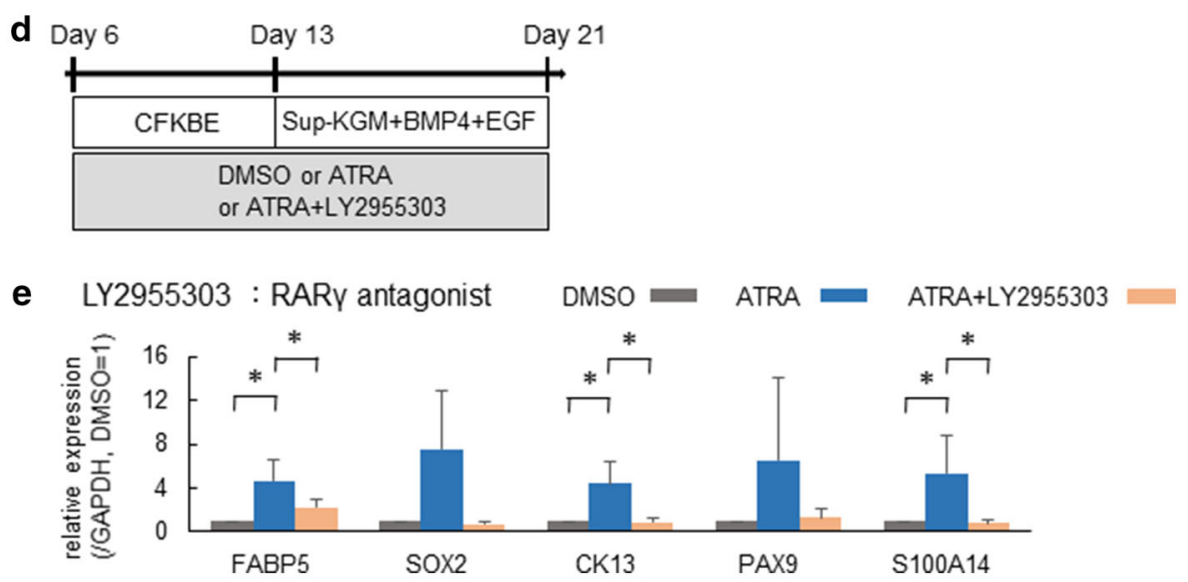

In Figure 3 caption, “(d, e )" should be corrected to "d, e"; "(g)" should be corrected to "g". In Figure 4 caption, "(b, $<\mathrm{BR}>\mathbf{c})$ " should be corrected to "b, $\mathbf{c}$ "; "(f)" should be corrected to "f".

In the "Abstract", the first sentence under the "Methods" section should read as "We established a novel stepwise protocol with transwell culture and an air-liquid interface system for esophageal epithelial cell (EEC) differentiation from hiPSCs."

In the Introduction section, the "*" should be deleted in the sentence "For example, several reports using *humaninduced stem cells" ... in second paragraph. In third paragraph, in the second sentence "retinoid acid" should be corrected to "retinoic acid".

In "Methods" section, under the "RA receptor-specific and antagonists" subsection "To assesses" should be corrected as "To assess" and "AM580 (Cayman Chemical, Ann Arbor, MI, USD)" should be corrected as "AM580 (Cayman Chemical, Ann Arbor, MI, USA)".

In "Methods" section, under the "Semi-quantitative or real-time quantitative reverse transcription polymerase chain reaction (RT-PCR)" subsection, "Trizol" should be corrected to "TRIzol" in the first line.

In "Methods" section, under the "Immunocytochemistry" subsection, the last sentence should read as "Hoechst33342 (WAKO) was used for nuclear staining."
In "Methods" section, under the "Frozen section samples" subsection in line 3, " -80 " should be corrected to "-80".

In "Methods" section, under the "RNA sequencing" subsection in line 6, "Novaseq6000" should be corrected to "NovaSeq6000" in the second sentence.

In "Results" section, under the subsection "Induction of EECs from hiPSCs", an improper line break should be deleted in the second paragraph and the line should read as "... during DE induction $[15,19]$, we examined the expression ...".

In "Results" section, under the subsection "Expression of esophageal marker genes in the differentiated cells with ALI culture" in line 13, "We, therefore," should be corrected to "We, therefore,".

In "Results" section, under the subsection "Enhancing effects of ATRA on esophageal epithelial differentiation", "SFTEB" should be corrected as "SFTPB" in the first sentence of third paragraph. In addition, the "ATRA $(+)$ " should be corrected as "ATRA $(+)$ " in the second and third paragraphs.

In "Discussion" section, the fourth sentence in the second paragraph should read as "One of them did not use any RA signaling agonists at all in their protocol [7]." The fourth sentence in the fourth paragraph should read as "Regarding the differentiation of skin, which highly express RAR $\gamma$ (S-Fig. 7a), ...”. 